\title{
A Model for Improving the Quality of Student Internship Placements in Engineering Degrees
}

\author{
doi:10.3991/ijac.v4i1.1518 \\ Rafael Garcia and Jordi Puig \\ University of Girona, Girona, Spain
}

\begin{abstract}
Work Internship Placements $(W I P)$ is a new and transversal enterprise internships programme, which is focused on quality improvement, academic control and satisfaction of collaborating enterprises. This programme is addressed to the engineering students of the Polytechnic School at the University of Girona (UdG) in Spain. The fundamental WIP infrastructure combines a web-based intranet platform, that provides a complete set of WIP tools, with a protocol of procedures and tasks that are observed and followed at all internship stages by every participating agent, i.e. enterprises, students, coaching professors and administrative staff. Our new programme is centered on a broader, more holistic internship placement procedure than the traditional "career and academic goals" approach. The WIP programme has been found to be a valuable asset in addressing enterprise and student needs in the experiential project.
\end{abstract}

Index Terms - work integrated learning; engineering internship programme; quality placements; intranet web platform; internship coaching.

\section{INTRODUCTION}

Exposing engineering students to a real working environment provides the student with a complimentary and fundamental view about engineering, being a key component in the Bologna process. This work integrated learning (WIL) approach could be included in the concept of "WIL as preparation for future employment" in the set of types of WIL described in [1,5,7].

The quality of the practical placements is determined by the contents, duration, allotted credits and also by the quality of resources, the stage organization and the procedures of monitoring and evaluating the competences acquired by the students. As described in [4], the success of a practical placement model requires the design and accomplishing of a quality assurance system for the practical stages, which comprises: (1) establishing objectives and quality assurance policies, (2) establishing organizational structures and responsibilities, (3) drawing up documents and human resources assurance, (4) monitoring, analyzing and improving the quality of the educational process through practice stages.

There are no general patterns for elaborating a quality assurance system for practical placements, since their structure is influenced by the general management of the university, by the philosophy of the university regarding the place and role of the practical placements, by economic and politicalsocial factors both at national and international level. Whatever the adopted system is, in order to be efficient and functional, it must attract the participa- tion of the whole staff and promote a new system of values in the university and in the company, specific to the quality culture, which includes continuous improvement, focus on clients, extended cooperation and partnership.

Enterprise internships have always had a great importance and interest in the bachelor engineering programs of the Polytechnic School (EPS) of the University of Girona (Spain). This preliminary professional training experience, carried out during the last academic year of the engineering studies, provides students with an opportunity to apply what they have learnt in a real environment and to acquire useful and efficient new work habits. Moreover, internships become a fundamental key factor in the student academic motivation, as also pointed out in [2].

Although the Polytechnic School understood internships as a key component of the academic curricula, some drawbacks were detected in the existing implementation of our internship programme. First of all, there was a lack of control of the working plan, due to a strong dissociation between the University and the enterprises. The coordinator of the engineering degree was formally responsible for the evaluation of the internships, among the many other tasks he had to carry out. Therefore, we could not effectively audit the academic contents of the internships. It should be noted that the coordinator's assessment was based only on a "pass/don't pass" strategy, and the student obtained a positive evaluation as far as he could prove that he had carried out the internship. Unfortunately, the university did not have a catalogue of companies offering internships. Therefore, the student had to look for a company, and the first contact between the university and the company was carried out by the student itself. With this situation, the offers could be too balanced towards the particular interests of either the enterprise or the student. Thus, in some cases, companies proposed working plans that were too biased towards their needs, sometimes without a valuable academic content for the student. The other undesirable situation with the existing internship model was that some students reached agreements with small companies were they had a personal/family contact, so that, in those cases, the student could not be objectively evaluated by the company. Finally, we had confirmed some cases of dissatisfaction with students regarding the academic content of their working plan, and with companies that were also disappointed because they were hiring students with the wrong profile and/or motivation.

For this reason, we started in 2005 a new transversal program of EPS internships that we named Work Internship Placements (WIP) which has been designed specifically to overcome the drawbacks of pre-existent programs while taking profit of their positive aspects. Thus, WIP is focused on internship quality improvement, increase of 
the academic control and, simultaneously, on promoting the interest and satisfaction of collaborating enterprises.

One of the key elements in the success of WIP is that it makes available to all internship participating agents (students, enterprises and university staff) a specifically designed intranet web platform which centralizes the set of tools, procedures and tasks involved in all internships stages: fluent communication among participants, partner contacts, information, registration, management, statistics and evaluation.

\section{WIP METHODOLOGY}

Before the deployment of WIP, the methodology to audit student internships was based on awarding some academic credits that were proportional to internship time. In contrast, in this new programme, students are enrolled in a specific WIP course - which is included in the engineering curriculum- having a selected team of coaching professors devoted to follow the advances of the student during the internship. A key point of the WIP programme is the adequate selection of these coaching professors, who not only monitor the internship, but also actively counsel students involved in this off-campus learning experience. For this reason, coaching professors should have experience in working with private companies, but should also know and understand the "culture" of every enterprise where a student is placed, guiding the student not only in the technical aspects of his work, but also in the typical way of behaving within the organisation. On the other hand, beyond providing support and guidance to the students, coaching professors also participate in the design of the internship activities, providing feedback to the companies and enabling synergies with existing research groups of the university that could add value to the company through technology transfer contracts. Therefore, adequate selection of coaching professors is one of the pillars for the success of the WIP programme. Moreover, in our programme, coaching professors get a reduction of their lecturing hours to facilitate this supervising activity. Because of this reduction in their lecturing hours, two additional part-time lecturers need to be hired to cover these courses. Thus, this programme requires some funding but, as described later on in section $\mathrm{V}$, the turnaround is worth this financial effort.

The WIP programme works as follows: (1) participating entreprises and institutions propose online their in situ engineering placements, (2) placement proposals are revised and approved by the WIP administration, (3) students access the web and apply for placements according the their curriculum and interests, (4) enrolled enterprises automatically receive an email with a link to examine the curriculum of the applicant every time a student selects that offer. Once the enterprise receives all the applications, the students are interviewed and, possibly with the help of coaching professors, the enterprise carries out its selection. (5) Whenever a student has been selected for a given placement, a coaching professor from EPS and an engineering coach from the entreprise are assigned to the student. (6) The internship starts with a meeting between the coaching professor, the enterprise coach and the student. During this meeting a working plan is defined in detail, pointing out the tasks that will be carried out by the student. (7) During the internship, those three partners are in contact at regular arranged times under the supervision of the coaching professor. (8) After the internship, the student is assessed by the coaching professor taking into account the enterprise opinion (this is carried out by contacting the enterprise coach).

\section{WIP WeB PlatForM}

The WIP intranet Web Platform (WIPWP) has been designed, implemented and configured specifically by the $E P S$ to satisfy the requirements of the WIP model (see Fig. 1). From a technical viewpoint, the platform has been always hosted on a Red Hat Linux server of our faculty.

WIPWP has been developed mainly in PHP language while some user interacting functionalities had been implemented in Javascript and AJAX. WIPWP is supported by a MySQL independent database and performing temporary data connections with other university central databases for data interchanging.

Despite its faculty specificity, WIPWP design easily allows being adapted and configured to be used by other faculties either in our university or outside. For instance, at present, and due to the success of the WIP model, the Faculty of Economics of our university is using its own adaptation of our WIPWP.

Functionally and from the user point of view, WIPWP has been split into five "agent" zones corresponding to the actors playing a role in the WIP model: public-common zone, enterprise zone, student zone, coaching professor zone and administrative staff zone. Moreover, there is a "super-agent" zone of administration-managing.

The platform works always under HTTPS network protocol to provide users a secure data flow. Access to the registered "agent" zones is protected by means of user defined passwords and specific PHP authentication code, while the "super-agent" zone uses a particular HTTP authentication scheme.

\section{A. Public-Common zone}

This area is the public unregistered zone of the intranet and also the common login door to "agent" registered zones. Its main functionality is allowing the registration of new enterprises to the platform; but it contains also general information about the WIP performance, the list of coaching professors, the list of collaborating enterprises and the public news section.

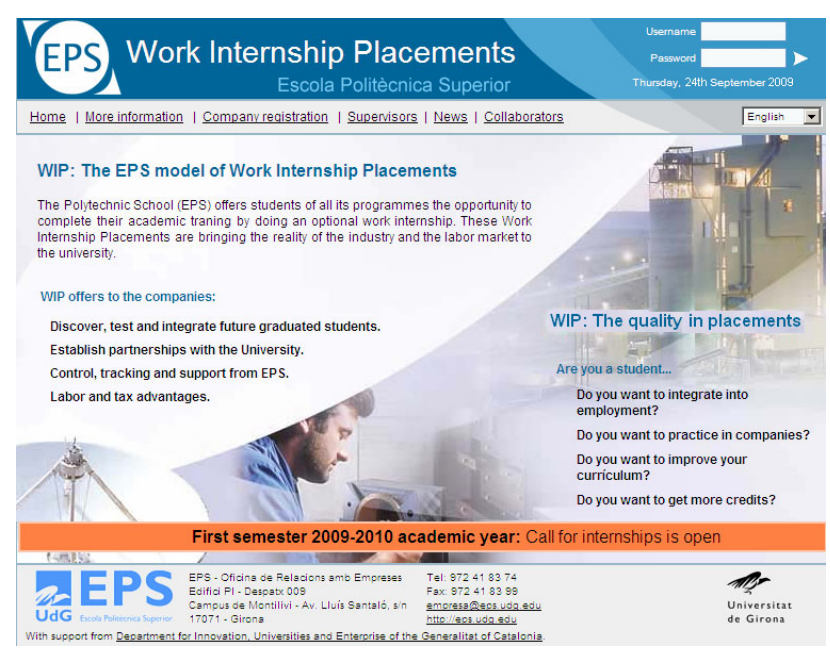

Figure 1. WIP homepage. Public zone which main functions are enterprise registration and registered-user login. 
Whenever an enterprise has filled in the registration form it receives an email message to verify its email address. To verify the confidence of those registrations, the WIP platform administrators contact all new registered enterprises at regular intervals or whenever a new enterprise makes a placement offer.

\section{B. Enterprise zone}

As already stated above, enterprises require a compulsory registration to access this zone which is dedicated to participating companies (see Figs. 2, 3 and 4).

The enterprise zone includes the following enterprise devoted services:

- Offers service. It includes: adding offers, state of offers, full offer information, cancellation of offers, list of applicants for every offer, access to curriculum of the applicants, etc.

- Selection service. Includes: selection of applicants setting the preference order, interview information, etc.

- Enterprise data service. Data modification of every enterprise in the platform.

- Coaching professors service. The list of coaching professors (including contact information) in every EPS degree.

- There is also a news and documentation section, a collaborators section and a help section.

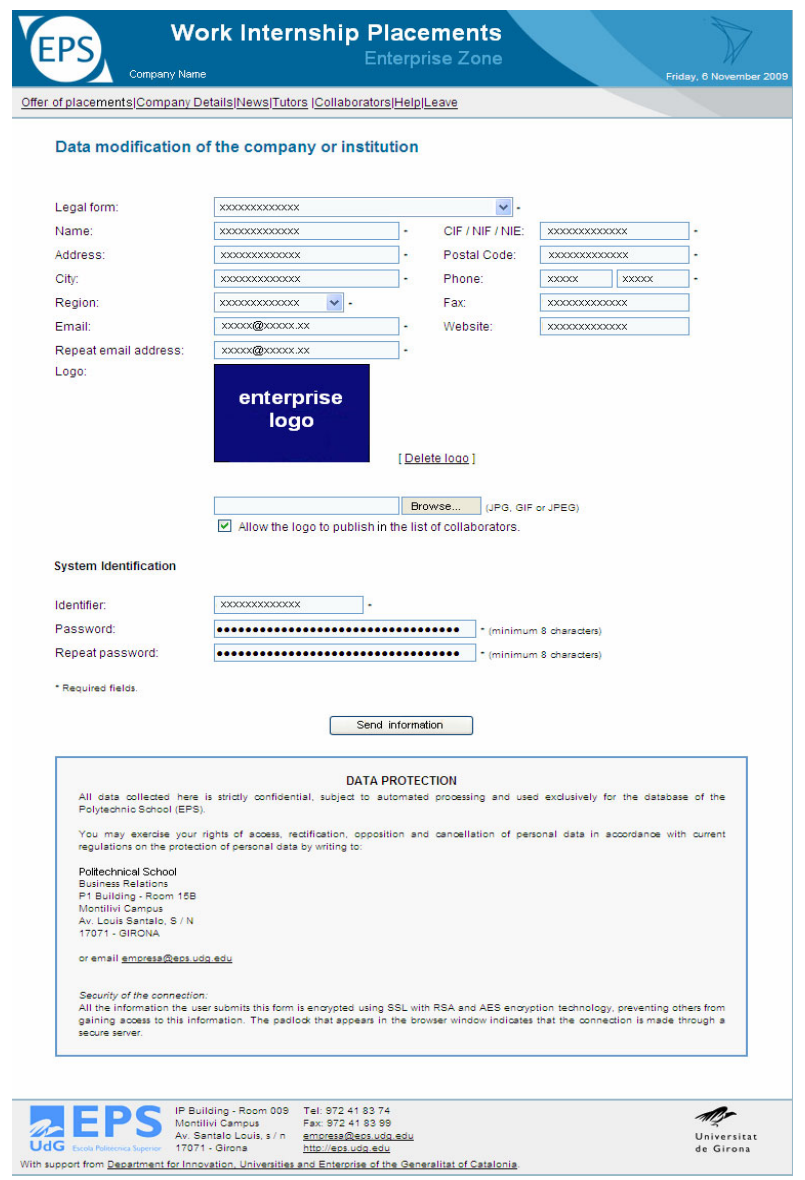

Figure 3. Sample web interfaces once the enterprise has registered online: Enterprise modification data form.

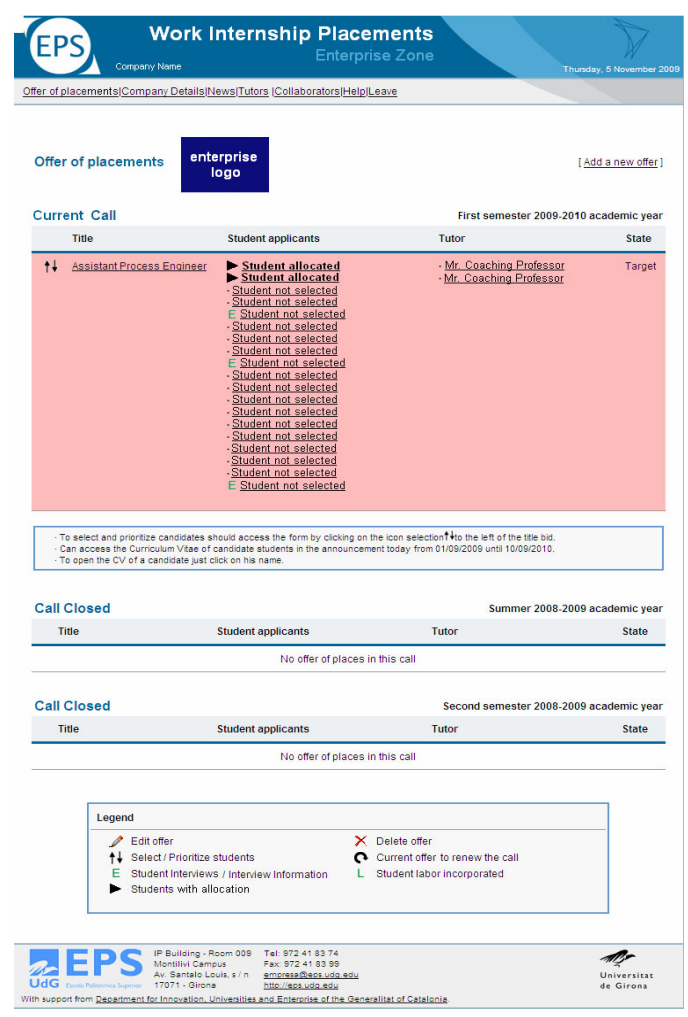

Figure 2. Sample web interfaces once the enterprise has registered online: Information about the offers submitted by an enterprise.

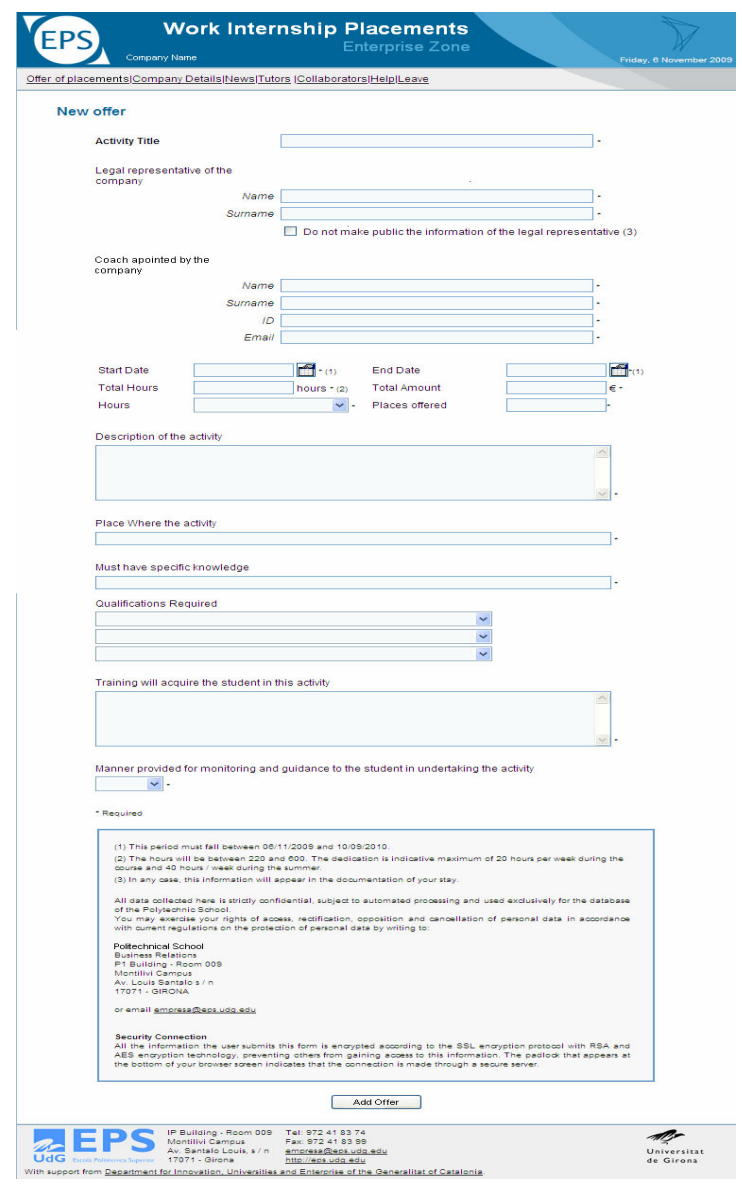

Figure 4. Sample web interfaces once the enterprise has registered online: Enterprise placement offer form. 

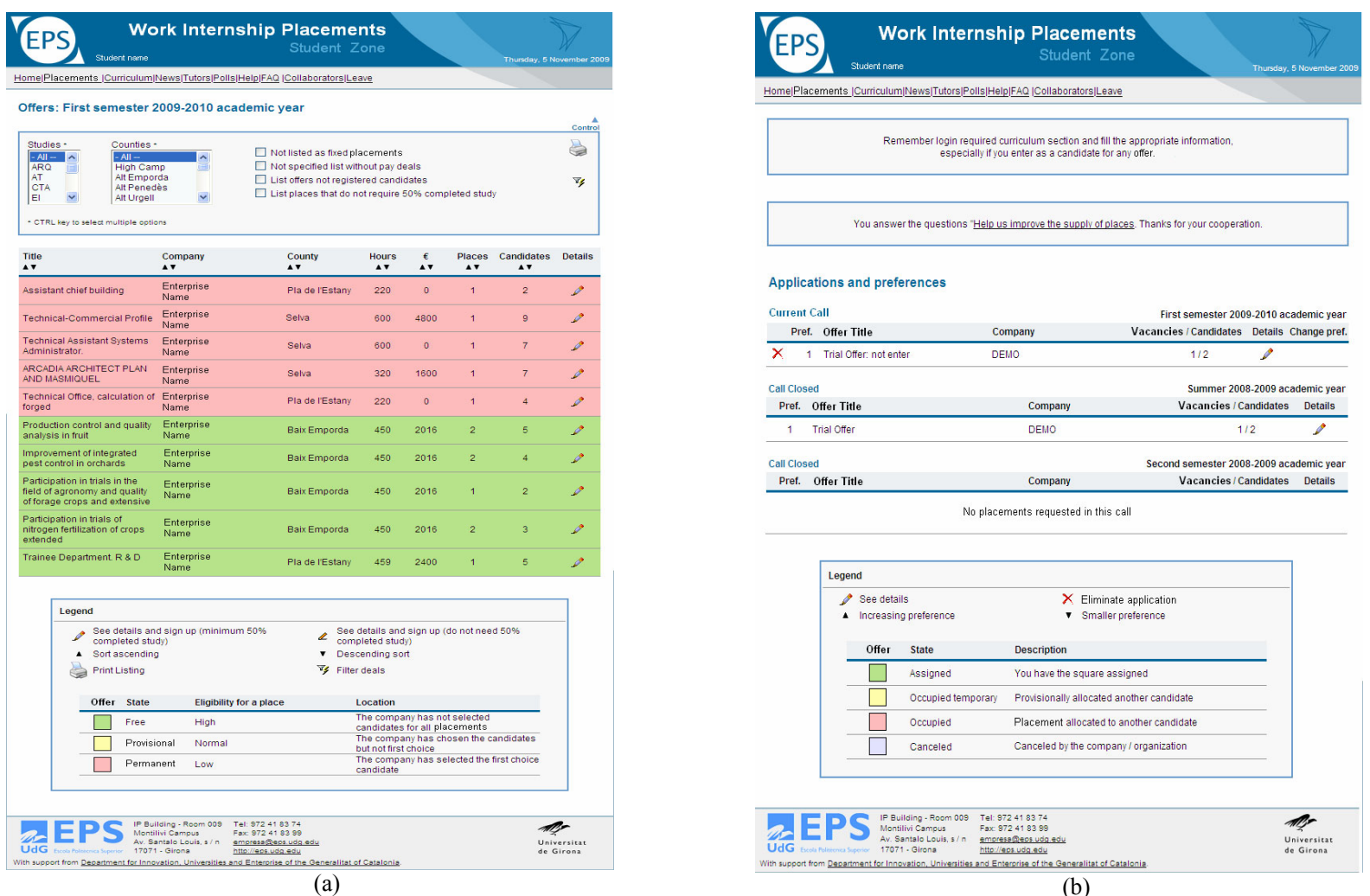

(b)

Figure 5. Student zone: (a) List of available placements seen by a student. (b) A student application form.

\section{Student zone}

Students, as members of the university community are already registered users of the university network, so EPS students do not need any specific registration in the WIP intranet and they should simply use their corresponding university intranet authentication data to access also our platform (see Fig. 5).

The student zone includes the following student dedicated services:

- Application service. It includes: state of allocation, full application information, cancellation of applications, change of preferences, etc.

- Curriculum service. The personal data and EPS training data are automatically filled in, so the student needs to complete only his/her work experience and additional information.

- Placements service. The complete list of placement offers (details, vacancies, number of applicants, state of allocation, etc.) which can be filtered depending on student interests and the application form.

- Coaching professors service. The list of coaching professors (including contact information) in every EPS degree.

- As in the enterprise zone there is also a news and documentation section, a collaborators section, a help section, a FAQ section and a surveys section.

\section{Coaching professor zone}

As explained before in the case of students, specific registration for WIPWP professors is not required.

The coaching professor zone includes the following services (see Fig. 6, 7 and 8):
- Coaching service. Including: list of assigned student-enterprise pairs, partner contact information, assess form, placement documentation state, etc.

- Enterprise service. Complete list of enrolled enterprises with complete contact information.

- Placements service. Complete list of placement offers (details, vacancies, number of applicants, state of allocation, etc.) which can be filtered depending on user interests.

- Coaching professors service. List of coaching professors (including contact information) in every EPS degree.

- It also includes: a news and documentation section, a collaborators section, and a help section.

\section{E. Administrative staff zone}

Administrative staff members are also users of the university network, so they do not require registration either. This zone is devoted to administrative staff services focused on providing information (list of placement offers, list of enrolled enterprises, list of coaching professors, administrative staff news, etc) and not on acting on the platform.

\section{F. Administration-Managing zone}

This zone is devoted to administrative and managing tasks of the WIP model and de managing utilities of the $W I P$ web platform itself.

The administration-managing zone includes the following detailed and extensive management services: enterprises, placements, applications, students, allocation algorithm, coaching assignments, coaching professors, placement documentation, emails, news, call for offers, statistics, etc. 

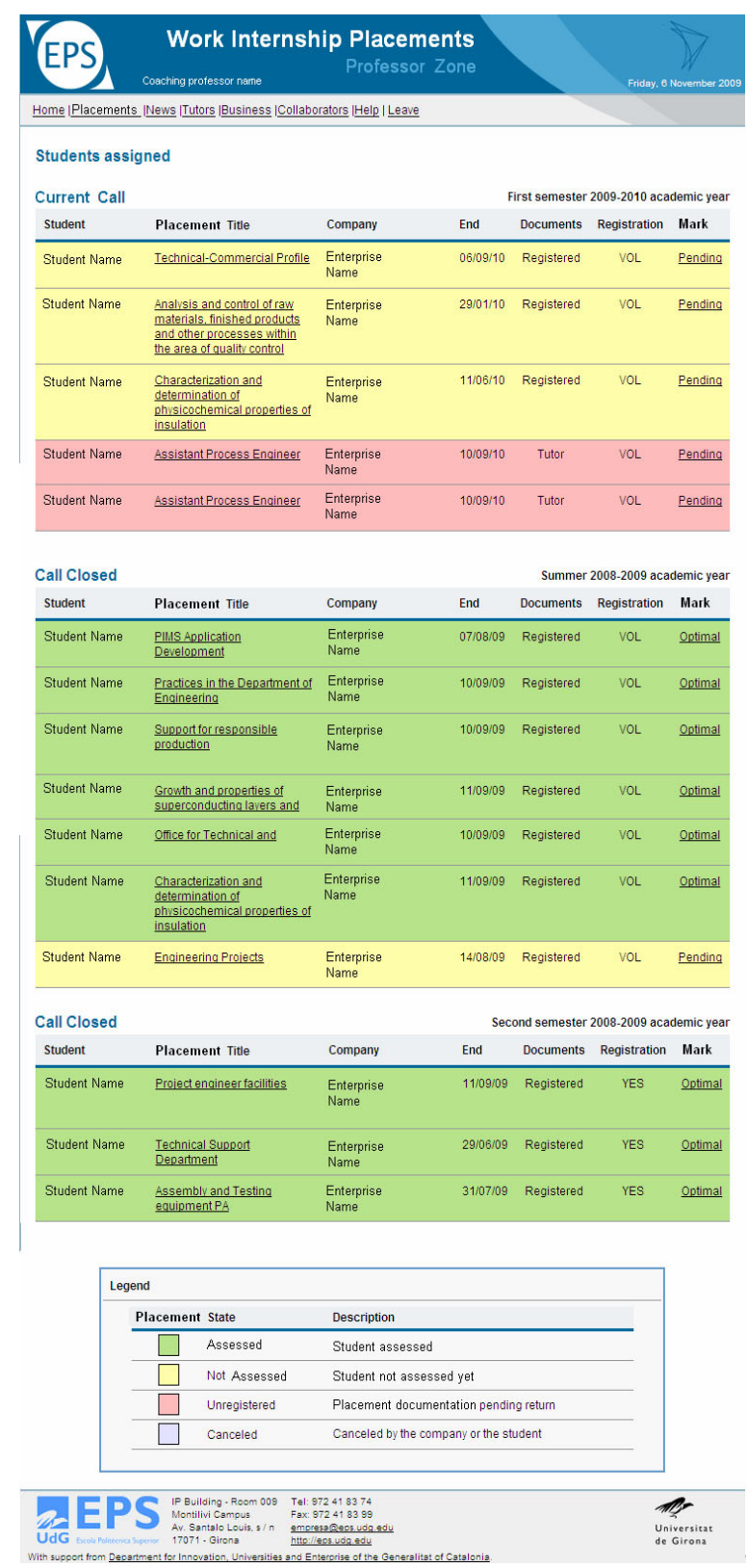

Figure 6. Coaching professor zone: List of students allocated to a coaching professor.

An interesting and useful functionality implemented in the "super-agent" zone is the possibility to emulate a login playing the role of any registered "agent" of the WIPWP. That login emulation is complete, in the sense that the "super-agent" could, not only monitor exactly what the emulated "agent" would see, but also act, if necessary, as the emulated "agent" itself. This emulation has several benefits: (1) instantaneous and exact monitoring of user's activities from their point of view, (2) efficiency in user problem solving, (3) executing actions on user demand, (4) testing new functionalities and (5) easiness of implementation by means of the correspondent "agent" zone services.

\section{WIP AllocAtion AlgORITHM}

Finally, WIPWP includes also an Automatic Allocation Algorithm (WIP3A) which, taking into account the declared preferences of the allocation's phase actors (place-
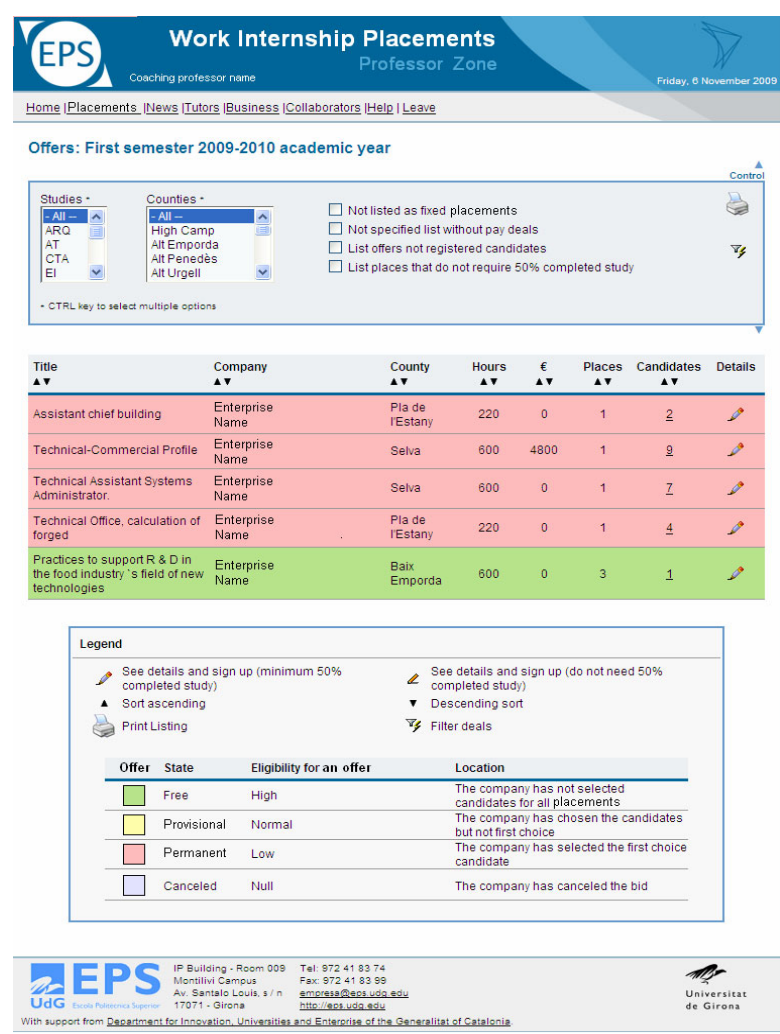

Figure 7. Coaching professor zone: List of placements as seen by a coaching professor.

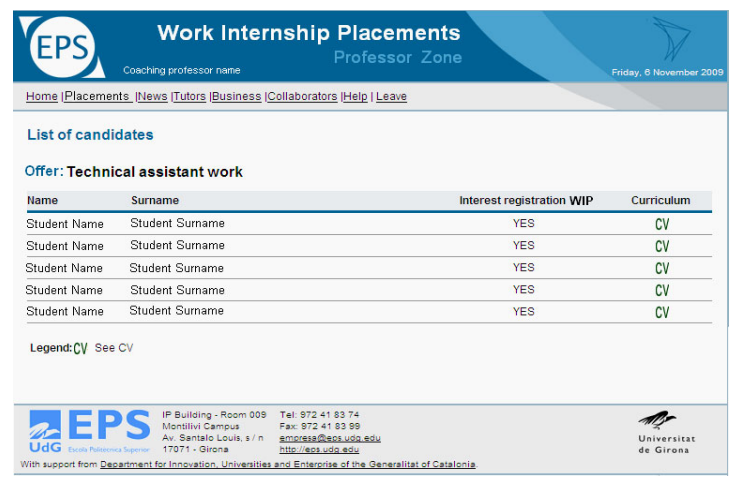

Figure 8. Coaching professor zone: List of applicants for a given placement as seen by a coaching professor

ment offering enterprises and applicant students) performs the allocation.

From the WIP3A point of view, students can be in one of these three different allocation states:

- Not allocated, when the student does not have an allocation.

- Provisionally allocated, when the student has an allocation which he has not yet accepted, so that allocation could be replaced by other student's priority allocations.

- Permanently allocated, when the student has an allocation already accepted by him or an allocation which has the highest student's appliance preference.

And placements can be in one of the following four different allocation states, according to the allocation algorithm: 
- Free allocation, the placement has no applicants or the algorithm has not set a different state to the placement yet.

- Void allocation, the placement does not have an allocated student after algorithm execution.

- Provisional allocation, whenever the placement has a provisionally allocated student.

- Permanent allocation, whenever the placement has a permanently allocated student.

WIP allocation proceeds as follows:

(1) Students can apply simultaneously to several placement proposals setting a preference order.

(2) Enterprises can also select several students from the corresponding applying offer list and set their preference order too.

(3) Every time $W I P 3 A$ is executed, placements in the void allocation state are set to the state of free allocation. Then the algorithm looks for the set of placements in state of free allocation, which is named the Free Placement Set (FPS). One free placement is taken from FPS and named the target placement -i.e. the placement that the algorithm would try to allocate- . Therefore, accordingly to the enterprise preference order, the first selected not allocated or provisionally allocated applicant in a lower student preference placement, if any, would be allocated in that target placement; otherwise -i.e. the target placement has no applicants, has only applicants permanently allocated or provisionally allocated in a higher preference placement- the placement is set to the void allocation state. Any new allocation would be always provisional excepting those cases where the target placement has the highest student preference or the student has already accepted it; only in these above mentioned cases the allocation would be permanent. The target placement would be always removed from the FPS. One of the collateral effects of this procedure will be that some provisional allocated placements could be let into a free allocation state and added to FPS, because its students "had been stolen" by other placements which are more interesting for those students. The process is repeated, by taking another free placement from FPS, until FPS is empty (i.e. there are no free placements left).

(4) WIP3A could be executed automatically whenever any agent carries out an action or in a managing supervised scheme at regular time intervals.

Finally, it must be noticed that despite the fact that both (applicant students and offering enterprises) had pointed out its preference order and enterprises had carried out a selection of students, the final decision (placement acceptation) and the prevailing preference criteria (for instance, whenever several enterprises had selected simultaneously the same applicant student) would be, obviously, on the student side.

\section{REsults}

The Work Internship Placements programme has currently completed its fifth year of operation. Table 1 summarizes the obtained results.
TABLE I.

QUANTITATIVE RESULTS FOR THE FIRST 5 YEARS OF THE WORK INTERNSHIP PLACEMENTS (WIP) PROGRAMME.

\begin{tabular}{|c|c|c|c|c|c|}
\cline { 2 - 6 } \multicolumn{1}{c|}{} & $\mathbf{0 5 / 0 6}$ & $\mathbf{0 6 / 0 7}$ & $\mathbf{0 7 / 0 8}$ & $\mathbf{0 8 / 0 9}$ & $\mathbf{0 9 / 1 0}$ \\
\hline $\begin{array}{c}\text { Enterprises in WIP } \\
\text { platform }\end{array}$ & 45 & 100 & 195 & 230 & 280 \\
\hline Enrolled enterprises & 35 & 70 & 130 & 90 & 106 \\
\hline Placements & 76 & 122 & 213 & 151 & 177 \\
\hline Applicant students & 111 & 171 & 245 & 370 & 450 \\
\hline Applications & 174 & 288 & 529 & 869 & 1,169 \\
\hline Allocated placements & 37 & 55 & 90 & 78 & 88 \\
\hline Unallocated placements & 39 & 67 & 123 & 73 & 87 \\
\hline "Optimum" evaluations & 33 & 39 & 49 & 55 & 67 \\
\hline "Suitable" evaluations & 4 & 16 & 24 & 22 & 20 \\
\hline $\begin{array}{c}\text { "Unsuitable" evalua- } \\
\text { tions }\end{array}$ & 0 & 0 & 1 & 1 & 1 \\
\hline
\end{tabular}

It should be noted that although the number of enterprises that joined the programme has been increasing at a good pace, the number of student placements has also increased, but it decreased on the 2008/09 academic year. This decrease is explained because of the international economic crisis, which has especially affected several companies of our local industry. On the contrary, the number of students that participate in the programme has kept a constant growth, with 450 students on the last year, generating more than 1,150 applications. This compares to the 111 students of the first year that generated 174 applications (see Fig. 9).

One of the keystones that enables this growth is the efficient performance of our automated web service, which allows an optimal interaction between students, enterprises and academic staff. This web portal is scalable, and allows the increase of the number of internships and companies with a very limited overhead for the administrative staff.

On the other hand, we observe that the performance of the students is very good. Since the students are qualified on a 3-grade system, most of them obtain the "optimum" grade, a few obtain a "suitable" and a much reduced number of students do not obtain a positive evaluation of the internship (see Fig. 10). In this grading, coaching professors consult and take into account the opinion of the company before judging the work of the student. Finally, it should be noted that the allocation of placements works very well by automatically following the algorithm described in section IV.

It is also important to note that, currently, the programme is not pre-defined. On the contrary, it is constantly developing. Continuous interaction and dialogue between faculty and industry is the cornerstone of the internship programme. For this reason, the web service has dedicated zones to for interaction between the different participating agents (students, faculty and companies) to interact in an optimal way. Moreover, at the end of the internship, we collect the opinion and observations of the enterprise coach, the student and the coaching professor. This feedback is crucial to the success of the project and critical for adopting corrective actions in the benefit of the programme.

Finally, regarding the coaching professors devoted to follow the advances of the student during the internship, 


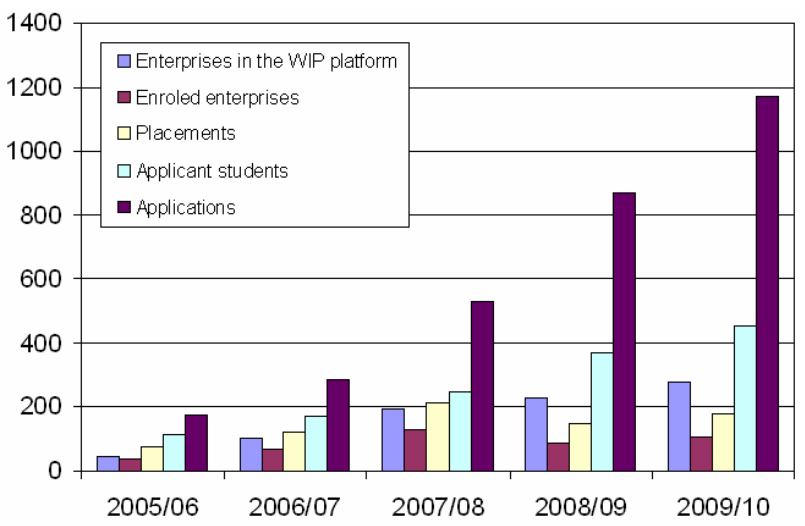

Figure 9. Evolution of the number of participating enterprises, internship offers, student demand, placements and evaluation results, for the first 5 years of the Work Internship Placements (WIP) programme.

we have observed two interesting aspects. First, the satisfaction of the companies for their active participation in the design of the internship activities (the reduction in their teaching load is a good motivation to increase their involvement). But also, by establishing links between industry and the existing research groups of the university, coaching professors are generating synergies that have become reality through an increase of technology transfer contracts. This increase in university-enterprise contracts compensates for the financial effort of hiring two additional part-time lecturers to cover the courses for which coaching professors get a reduction. For all these reasons, and provided that coaching professors are a key element in the success of the project, it should be noted that efforts are also being made to obtain new faculty members with industrial experience who will eventually act as coaching professors.

\section{CONCLUSIONS}

Currently, the WIP course is not yet mandatory in our degree programmes, but according to the strategic guidelines of the Bologna process and as a result of its success, it will be mandatory in the near future for some of engineering degrees of EPS. This mandatory nature of the WIP is also supported by the regional environment business world main opinion that this course makes a very significant contribution towards providing industry with engineering graduates who are well-prepared to assume responsible and productive engineering assignments, with less need for on-the-job training and with generic skills through problem-based learning (as described in [6]). This significant contribution agrees with many research works, e.g. the UK study presented in [3], where the author points out the positive effect of industrial placement schemes on future engineer students employability. For all these reasons we conclude that this preliminary professional training experience, obtained through enterprise internships and surveyed by a motivated coaching professor, is a fundamental key factor in the student learning process.

Moreover, it should be noted that the development of a web platform that automates and monitors the different steps of the internship process is a key tool to improve the quality both for the students and for the enterprises. This model is perfectly scalable, so that an increase of the number of internships carried out by the students of the

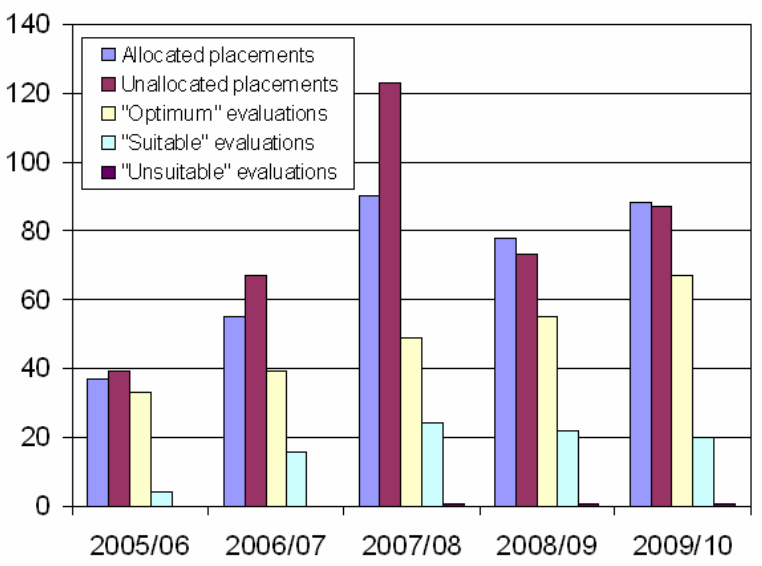

Figure 10. Number of placements and evaluation obtained by the students for the first 5 years of the Work Internship Placements $(W I P)$ programme.

Polytechnic School can be easily handled by the web platform.

We should remark that both enterprise and student feedback has been overwhelmingly positive for WIP, with a special emphasis in the functionality of the web application and the active participation of the coaching professors. Indeed, many of the participating companies regularly repeated his offer of placements. Moreover, the growing interest of students is clearly evident in the increasing number of candidates and their strong wish to repeat the WIP experience.

On the other hand, one of the most important lessons that we have learnt is that the adequate selection of the coaching professors, with solid experience in working with companies and industries, is key point for the success and positive turnaround of the programme for students, companies and also research groups of the university.

Finally, it should be noted that we see many cases where, from a practical internship, the link between student and company continues and formalizes a contract of employment once the student completes his degree in engineering. Thus, work internships not only help to supplement the practical training of students but are also an excellent tool for future job placement.

For all the reasons stated above, it is important to develop scalable web-based intranet platforms that provide tools, procedures and tasks to manage student internships at all stages, providing feedback not only to students but also to enterprises, coaching professors and administrative staff.

\section{VIII.FUTURE WORK}

The future main project related to WIP is the development of an automated WIP Survey Web Platform (SWP). The aim of $S W P$ is obtaining useful and valuable feedback from the WIP participating actors (mainly enterprises and students) to improve the quality of the general WIP model in all its features, i.e. information, security, functionality, efficiency, etc. This survey platform, which at this moment is in a design and initial development stage, would finally be integrated in the general WIPWP project.

In parallel, the web platform will continue its process of evolution, continuous improvement and increased functionality, adapting to the needs and suggestions of different user groups. 


\section{ACKNOWLEDGMENT}

This project has been partially funded by the Catalan Government through project 2007MQD00163. The authors would also like to thank the Polytechnic School $(E P S)$ of the University of Girona for their support.

\section{REFERENCES}

[1] J. Brennan, B. Little "A Review of Work Based Learning in Higher Education” London: DfEE, 1996.

[2] D.A. Colley, D. Jamison, "Postschool results for youth with disabilities: Key indicators and policy implications" Career Development for Exceptional Individuals, 21(2), 2008, pp. 145-60. doi: $10.1177 / 088572889802100204$

[3] D. Hall, "Industrial Placement - Preparation for Success". Available online from: http://www.science-engineering.net/ /placement g.htm $>$ [Access: September, 2010].

[4] E. Helerea, M. Popescu, L. Bădescu, A. Dascălu, "Management System for the practical placement in higher education". In D. Talabă (coord.) European Models and Best Practice on Practical Placements of Students. Proceedings of the 3rd. EUI-Net International Workshop, Rome, 2007, pp. 87-90.

[5] E. Martin, "The Effectiveness of Different Models of Work-based University Education" Department of Employment, Education, Training and Youth Affairs J.S. McMillan Printing Group, 1997.

[6] L. Uden, G. Moran, "More than subject-specific skills for students". In D.Talabă. (coord.) European Models and Best Practice on Practical Placements of Students. Proceedings of the 3rd. EUINet International Workshop, Rome, 2007, pp. 51-56.

[7] I.T. Wangsa, L. Uden, "Towards the development of industrial placement in Higher Education”. In D. Talabă. (coord.) European Models and Best Practice on Practical Placements of Students. Proceedings of the 3rd. EUI-Net International Workshop, Rome, 2007, pp. 9-14.

\section{AUTHORS}

R. Garcia is vicedean of the Polytechnic School and head of the Computer Vision and Robotics group of the University of Girona in Spain. Since 2004 Dr. Garcia is responsible of the Enterprise Relations Office of the Polytechnic School (e-mail: rafael.garcia@udg.edu).

J. Puig is with the Enterprise Relations Office of the Polytechnic School, University of Girona, Girona 17071 Spain. Since 2005 Mr. Puig is the coordinator of the WIP programme, development responsible and webmaster of the WIP intranet platform (e-mail: j.puig@udg.edu).

A preliminary version of this paper was presented in the IEEE EDUCON 2010 conference and selected for submission to this journal. It received an award in the Knowledge and Competencies in Engineering area of that conference.

Submitted November $20^{\text {th }}, 2010$. Published as resubmitted by the authors January $27^{\text {th }}, 2011$. 